\title{
Sistemas de protección primaria para daguerrotipos
}

\author{
Clara M. Prieto
}

Resumen: Los sistemas de protección para daguerrotipos han sido, y siguen siendo, una de las principales preocupaciones de sus productores originales y de sus custodios actuales. Los sistemas de protección primaria son esenciales para garantizar la preservación de los daguerrotipos: proporcionan estabilidad física y química a la placa daguerriana y minimizan los efectos que los agentes de deterioro pueden provocar en ella. Los materiales que conforman este sistema de protección constituyen la primera barrera que protege al objeto y pueden, en gran medida, modular las fluctuaciones de humedad relativa y filtrar los agentes contaminantes presentes en el ambiente. En este artículo se ofrece una revisión ordenada de la literatura científica específica sobre los sistemas de protección primaria para daguerrotipos, analizando los materiales empleados y los resultados obtenidos.

Palabras clave: daguerrotipo, preservación, sistema de protección primaria, embalaje, almacenamiento, materiales

\section{Primary housing systems for daguerreotypes}

Abstract: Daguerreotype housing systems have been, and still remain, one of the main concerns of its original producers and their current custodians. Primary housing systems are essential to ensure the preservation of the daguerreotypes: they provide physical and chemical stability to the daguerrean plate and minimize the effects of the agents of deterioration on them. The materials that constitute the primary package set up the first barrier for the object protection, so as they can modulate fluctuations in relative humidity and can filter the pollutants present in the environment. This article provides a methodical review of specific scientific literature on this subject, analyzing the materials used and the results obtained.

Key words: daguerreotype, preservation, primary package, housing system, archival, storage, materials

\section{Sistemas de proteção primária para daguerrótipos}

Resumo: os sistemas de proteção para daguerreótipos têm sido, e continuam a ser, uma das preocupações principais dos seus produtores originais e dos seus depositários atuais. Os sistemas de proteção primária são essenciais para garantir a preservação dos daguerreótipos: proporcionam estabilidade física e química à placa e minimizam os efeitos que os agentes de deterioração podem provocar. Os materiais que constituem este sistema de proteção constituem a primeira barreira que protege o objeto e podem, em grande medida, modular as flutuações de humidade relativa e filtrar os agentes poluidores presentes no meio ambiente. Este artigo oferece uma revisão ordenada da literatura científica espanhola sobre os sistemas de proteção primária para daguerreótipos, analisando os materiais empregues e os resultados obtidos.

Palavras-chave: daguerrótipos , preservação, sistema de proteção primária, embalagem, armazenamento, materiais 


\section{Introducción}

Un daguerrotipo es una imagen directa, única y frágil formada por una amalgama de plata y mercurio depositada sobre la superficie especular de una placa de cobre plateada. Según el ángulo de observación del espectador aparece como un positivo o un negativo. Cuando esta imagen única era juzgada como buena por el fotógrafo y su cliente, se la dotaba de un montaje de presentación/preservación a la altura estética de la preciosa imagen contenida. Según las costumbres, usos y modos de vida propios de cada país, estos sistemas de presentación podían ser un montaje en passepartout o marco decorado, o un estuche. La mayor parte de los daguerrotipos que han llegado a nuestros días ha sido gracias a su montaje. Este constituye una protección a la vez química y física frente a los agentes de deterioro, evitando los daños en la imagen por impactos, arañazos, limpiezas bienintencionadas o los ya bien conocidos efectos de los contaminantes ambientales sobre la plata.

Son numerosas las publicaciones acerca de la técnica fotográfica del daguerrotipo, así como aquellas dirigidas a coleccionistas y daguerrotipistas que, sin duda, han influenciado los métodos de montaje que se utilizan en el campo de la conservación de fotografía.

En el presente trabajo se analizan los sistemas de protección primaria para daguerrotipos aplicados desde los años 70 hasta la actualidad. Asimismo, se ha elaborado una tabla resumen con los detalles técnicos relativos a los materiales y productos que han sido empleados en dichos sistemas de protección [Tabla 1]. A modo de conclusión se presentan los resultados del estudio, así como un análisis del estado de la cuestión.

\section{Sistemas de protección}

Es importante considerar los sistemas de protección como estructuras multicapa, desde el nivel de protección más directo, aquel que está en contacto directo con el daguerrotipo, hasta el más exterior, el que va a estar en contacto con el usuario del mismo. Con el fin de establecer una terminología precisa se definen los siguientes términos y se ilustran con los esquemas recogidos en la Figura 1:

-Términos relacionados con la producción del daguerrotipo (1839-1860):

- Placa daguerriana.- positivo directo de cámara sobre soporte de metal (plata o cobre y plata) y cuya imagen final está formada por una amalgama de mercurio y plata (a partir de 1840, también oro).

- Paquete daguerriano.- protección primaria y original del daguerrotipo. Consta, como mínimo, de la placa daguerriana, un separador/espaciador de papel o metal y un vidrio de protección, todo ello sellado mediante una tira de papel engomado (Fuentes de Cía, 2004c).
- Sistema de sellado original.- Sistemas de sellado empleados para dotar de protección primaria a las placas daguerrianas en origen, en el momento de su obtención.

- Sistema de montaje original-Sistema de montaje empleado para la presentación del paquete daguerriano. Los formatos de presentación para daguerrotipos empleados en el siglo XIX se despliegan, principalmente, en dos modelos: el estuche (estilo americano) y el marco o passepartout (estilo europeo), existiendo otros como por ejemplo, joyas.

-Términos relacionados con la conservación del daguerrotipo (1970-actualidad)

- Sistema de protección.- Sistema destinado a la conservación del daguerrotipo, en general, aplicable en varios niveles $y$ a diversos artefactos ${ }^{1} \mathrm{y} / \mathrm{o}$ partes de los mismos. Housing system.

- Sistema de protección primaria.- Sistema destinado a proteger la placa daguerriana, que incluye todos los elementos protectores de la misma: trasera, espaciador, vidrio protector y sellado, excluyendo la forma de presentación final del daguerrotipo. Primary housing system. Primary package. Archival package (Murata 2003). Si se trata del montaje original será denominado paquete daguerriano.

- Sistema de protección secundaria.- Sistema diseñado para proteger al paquete daguerriano, o para contener al sistema de protección primaria. Secondary housing system. Preservation package (Wiegandt 2005).

- Sistema de protección terciaria.-Sistema diseñado para proteger al artefacto daguerriano en cualquiera de sus formatos de presentación original, generalmente para su almacenaje. Tertiary housing system (Murata 2003).

\section{Sistemas de protección primaria}

Los sistemas de protección primaria son aquellos destinados a proteger a la placa daguerriana.

El comienzo de los sistemas de protección primaria modernos puede ser datado en los años 70 del siglo $X X$, fecha en la que se inicia el desarrollo del campo profesional de la conservación de fotografía en un marco científico. Entre los años 60 y 70 se popularizaron los tratamientos de limpieza de placas daguerrianas, y fueron muy numerosos los daguerrotipos intervenidos en esta época. Esta práctica implicó la destrucción de los sellados originales, al mismo tiempo que se descuidó el resellado de las placas, lo que desembocó en una peligrosa aceleración de los procesos de deterioro de las mismas. 


\begin{tabular}{|c|c|c|c|}
\hline $\begin{array}{l}\text { PROTECCIÓN } \\
\text { NIVEL }\end{array}$ & Denominación & Original & Moderno \\
\hline \multirow{2}{*}{ Primario } & $\begin{array}{l}\text { Sistema de } \\
\text { sellado } \\
\text { (Daguerre 1839) }\end{array}$ & $\begin{array}{|lll|}\text { PAQUETE DAGUERRIANO } \\
\text { Vidrio } \\
\text { Sello } \square \text { Slaca daguerriana }\end{array}$ & \\
\hline & $\begin{array}{l}\text { Sistema de } \\
\text { protección } \\
\text { primaria } \\
\text { (GEH1970) }\end{array}$ & & \begin{tabular}{|c|c|} 
SISTEMA DE PROTECCIÓN PRIMARIA: \\
Sistema de protección de la placa daguerriana. \\
Primary housing system/Archival package \\
Vidrio \\
Placa daguerriana \\
Sello
\end{tabular} \\
\hline \multirow{2}{*}{ Secundario } & $\begin{array}{l}\text { Sistema de } \\
\text { presentación } \\
\text { (Desconocido } \\
\text { circa 1839) }\end{array}$ & 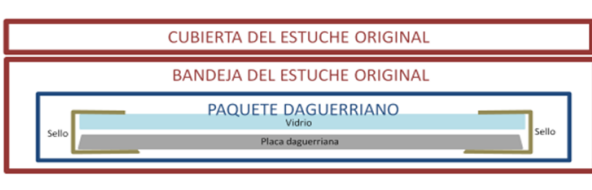 & \\
\hline & $\begin{array}{l}\text { Sistema de } \\
\text { protección } \\
\text { secundaria } \\
\text { (Wiegandt2005) }\end{array}$ & & 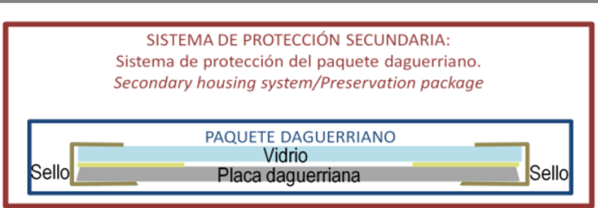 \\
\hline \multirow{2}{*}{ Terciario } & \multirow{2}{*}{$\begin{array}{l}\text { Sistema de } \\
\text { protección } \\
\text { terciaria } \\
\text { (Murata 2003) }\end{array}$} & & 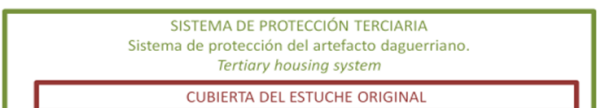 \\
\hline & & & 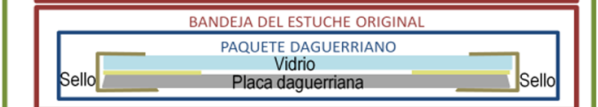 \\
\hline \multirow[b]{2}{*}{ Estructural } & \multirow{2}{*}{$\begin{array}{l}\text { Sistema de } \\
\text { protección } \\
\text { estructural } \\
\text { (Prieto2016) }\end{array}$} & & $\begin{array}{c}\text { SISTEMA DE PROTECCIÓN ESTRUCTURAL } \\
\text { CUBIERTA DEL ESTUCHE ORIGINAL }\end{array}$ \\
\hline & & & 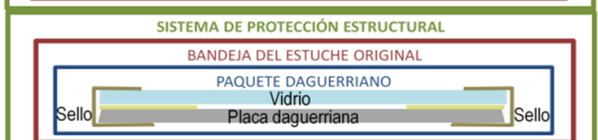 \\
\hline
\end{tabular}

Figura 1.- Esquema: sistemas de protección originales y modernos, según nivel de protección: primario, secundario y terciario, indicando primera referencia bibliográfica. Elaboración: ${ }^{\circledR}$ Clara M. Prieto.

Muchos coleccionistas, e incluso conservadores de la época, se dieron cuenta de la necesidad de "restaurar" los sellados de las placas y emplearon para ello un amplio abanico de productos comerciales, como cintas adhesivas tipo "cello", cinta aislante, cinta de enmascarar, cinta de carrocero, no resultando, en todos los casos, adecuadas para su función. En ese momento inicial del desarrollo de la profesión de conservador-restaurador de fotografía, los restauradores de documento gráfico aportaron las técnicas, los materiales y la práctica de la restauración de papel al campo de la fotografía. Para empezar, cuestionaron el uso generalizado de cintas adhesivas comerciales, cuyos efectos nocivos sobre los documentos y las obras de arte en papel conocían sobradamente. También se comenzó a poner en cuestión la calidad de los materiales empleados hasta ese momento para la elaboración de montajes de conservación para daguerrotipos.
En los años 70, la George Eastman House se enfrenta a la necesidad de dotar de un sistema de protección primario a las numerosas placas daguerrianas que albergaba en sus fondos, y diseña esta estructura, conocida en la institución como GEH\#1:

En este sistema se emplea papel Permalife ${ }^{\circledR}$ adherido con acetato de polivinilo (Elvace 1874) para sellar el conjunto de protección primaria paquete, pero se emplean cuatro tiras, una para cada lado, en lugar de una tira continua rodeando el conjunto, y el sellado no se extiende sobre el vidrio. Se incluía un espaciador de material celulósico, el mismo cartón de calidad "permanente" ${ }^{2}$ utilizado para la trasera.

La primera referencia que encontramos a un sistema de protección primaria, montaje de conservación moderno para daguerrotipos, en la bibliografía data de 1972. En el 


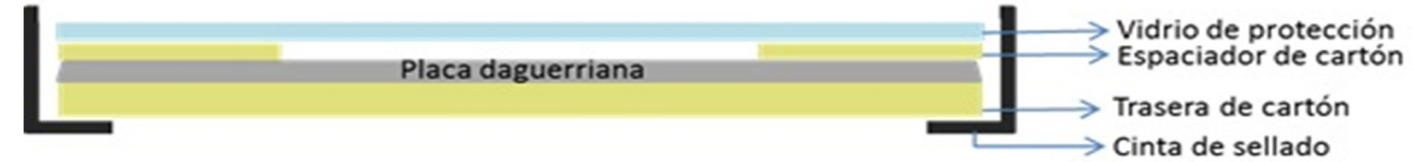

Figura 2.- Esquema del sistema de protección de la placa GEH\#1. Elaboración propia a partir de Murata (2003)

capítulo "Cleaning a blackened daguerreotype" del libro Caring for Photographs: display, storage, restoration (TIME LIFE BOOKS 1972), detalla instrucciones para desmontar el paquete daguerriano, pero no da indicaciones para volver a montarlo ni para sellarlo.

En 1976, Siegfried Rempel publica un artículo en el número 3 de Archivaria: The Journal of Canadian Archivist en el que no se describe método alguno para el sellado de las placas daguerrianas, pero que incluye, sin embargo, mención a una recomendación dada en comunicación privada por Robert Organ del Smithsonian Institute, en la que aconseja incluir un material que absorba las posibles emanaciones sulforosas entre el espaciador y la placa daguerriana, con el fin de neutralizar aquellas que pudieran penetrar en el estuche.

Robert A. Weinstein y Larry Booth, en su libro Collection, Use, and Care of Historical Photographs, publicado en 1977, describen un método para el sellado original de las placas daguerrianas, como parte de su proceso de producción, en el que se detalla que la placa se protegía con un marco de latón ornamentado y se cubría con un vidrio. Este "sándwich" se sellaba con una cinta de papel y se añadía entonces una orla o borde decorativo, llamado preservador. Este paquete se colocaba en un estuche articulado, ornamentado y dotado de un broche de cierre, en el caso de estuches pequeños, o de dos broches, en el caso de estuches de mayor tamaño. En el mismo capítulo se dan instrucciones acerca de cómo desmontar un daguerrotipo para su limpieza y tratamiento, advirtiendo, entre otros riesgos, acerca de la sensibilidad de la placa frente a la contaminación atmosférica e indicando la importancia de un correcto sellado de la misma para prevenir su ennegrecimiento. Los materiales recomendados para el sellado son papel Permalife ${ }^{\circledast}$ adherido con adhesivo de acetato de polivinilo (Jade ${ }^{\circledR}$ 403). En el mismo año se publica Shoots: A Guide to Your Family's Photographic Heritage (Davies 1977), en el que detalla instrucciones para el desmontaje de un daguerrotipo, pero no indica cómo volver a montarlo ni resellarlo. A este respecto, la revista Archivaria (Moore 1977) publica una crítica acerca de dicho libro, en la que se señala que está escrito para neófitos y que, aunque puede ser de utilidad "resulta desafortunado que muchas de las ideas no estén expresadas claramente y que sean a menudo incorrectas".

Alice Swan (1978), en su artículo “Conservation Treatments for Photographs: A Review of Some of the Problems, Literature and Practices", da a conocer el sistema de montaje practicado entonces en la George Eastman House y conocido como GEH\#2.

En el citado artículo se recomienda testar el pH y reactividad con la plata de los materiales empleados para el montaje, siguiendo los protocolos desarrollados

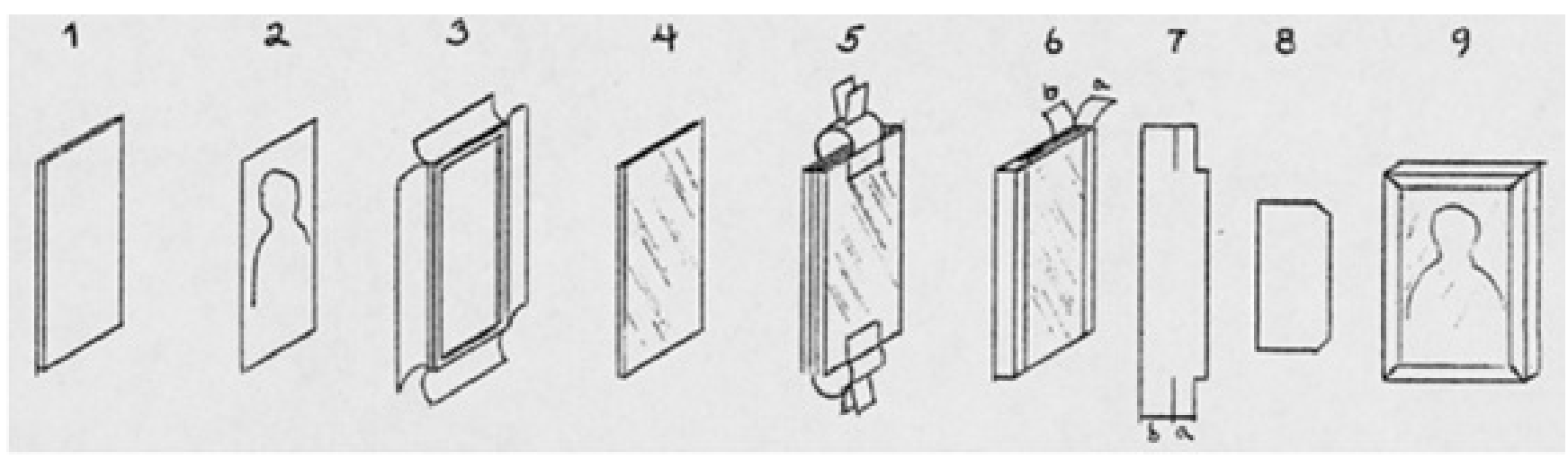

Figura 3.- Esquema del sistema de protección de la placa GEH\#2 (Swan 1978).1. Trasera de cartón Museo; 2. Placa daguerriana; 3. Espaciador estrecho de cartón Museo con solapas de papel Permalife ${ }^{\circledR}$. Las solapas se pliegan sobre los bordes de la placa daguerriana; 4 . Vidrio; 5. Sistema de protección ensamblado y sujeto con pinzas para facilitar su sellado: (piezas 1,2,3,4); 6. La cinta de sellado se aplica primero en los lados largos. La solapa (a) de la esquina se pliega y adhiere antes que la solapa (b), que se pliega y adhiere sobre la solapa (a); 7. Configuración de la pieza de cinta de sellado para los lados largos; 8. Configuración de la pieza de cinta de sellado para los lados cortos; 9. Sistema de protección completo con la cinta de sellado cubriendo los bordes. Traducción: Clara M. Prieto 
por Collins y Young (1976). Asimismo, enuncia que los montajes han de ser elaborados a partir de los materiales más seguros de los que se pueda disponer: con pH neutro, libres de azufre, papel de pasta de trapos 100\%, papel de calidad "permanente" y emulsiones inertes de acetato de polivinilo, sugiriendo el producto Elvace ${ }^{\circledR} 1874$.

Con el fin de completar el montaje de la colección de daguerrotipos de la Colección Southworth \& Hawes, en 1980, la George Eastman House implementa algunas mejoras en el sistema de protección primaria de las placas. El papel Permalife ${ }^{\circledR}$ adherido con acetato de polivinilo es sustituido por cinta adhesiva activada por presión, Filmoplast ${ }^{\circledR}$ P90, y el sellado se extiende sobre el vidrio, con el fin de mejorar la estanqueidad del sistema. Como quedó demostrado posteriormente en la investigación llevada a cabo por H. Murata (2003), la cinta de sellado contribuye a dotar al sistema de protección primaria de un entorno más estable cuando se extiende sobre el vidrio. Asimismo, esta investigación confirmó que dos capas de cinta de sellado protegían más que una, y que era mejor utilizar una tira continua para rodear al paquete que cuatro tiras separadas, una para cada lado.

En 1982 se publica la edición revisada del libro Caring for Photographs: display, storage, restoration (TIME LIFE BOOKS 1982), incluyendo la revisión del artículo "Cleaning a blackened daguerreotype" bajo el nuevo título "Cleaning a tarnished daguerreotype". En él se mencionan de nuevo instrucciones para el desmontaje del paquete daguerriano y la extracción de la placa, pero sigue sin indicar cómo montarlo de nuevo ni cómo sellarlo.

Dos años más tarde, en 1984, en el libro The life of a photograph (Keefe e Inch 1984) se sugiere el uso de Scotch ${ }^{\circledast}$ Magic $^{\mathrm{TM}}$ Transparent Tape o cinta de papel para el sellado de los daguerrotipos. Recomiendan papel japonés o "Permalife ${ }^{\circledR}$ bond", unido con un adhesivo de acetato de polivinilo, como Jade ${ }^{\circledR}$ 403. Para facilitar la aplicación de la cinta, recomiendan utilizar dos secciones y superponerlas en las esquinas, donde es necesario que proporcionen un sellado hermético. De nuevo se evidencia la preocupación por la estanqueidad del sistema.

Ese mismo año, en el libro Archives \& manuscripts-administration of photographic collections (Ritzenthaler, et al. 1984), se recomienda no eliminar los sellados originales, si estos están intactos. En el caso de que aparezcan dañados, recomiendan su sustitución por Filmoplast ${ }^{\circledR}$ P90, siguiendo el esquema detallado en la Figura 4.

Como sistema de protección primaria para placas daguerrianas sin protección recomiendan un montaje compuesto por un vidrio de protección, una trasera y un espaciador en soporte celulósico (cartón de pH neutro), todo ello al tamaño de la placa y sellado con Filmoplast ${ }^{\circledR}$.

En 1986 Barger, en su artículo "Cleaning daguerreotypes" del número 31 de Studies in conservation hace referencia al método $M R L^{3}$ de tratamiento de daguerrotipos, incluyendo instrucciones para el resellado del daguerrotipo ${ }^{4}$ mediante el uso de un espaciador, un vidrio moderno y cinta adhesiva de papel permanente ${ }^{5}$ activada por presión para sellar el paquete.

Es interesante la recomendación de no utilizar papel para cubrir el interior de los preservadores de latón, práctica que se venía realizando con la intención de evitar que el preservador arañara la placa. Los estudios de Barger concluyeron que el papel mismo podía arañar la superficie de la placa más que el preservador (si este ha sido convenientemente suavizado-lijado-mateado), además de introducir un nuevo agente de deterioro favorecedor de la corrosión: el papel incrementa el nivel de humedad en el paquete más de lo que lo hace el latón del preservador original. En el mismo artículo se indica que la medida más importante para la conservación de los daguerrotipos es asegurarse de que están protegidos con un vidrio nuevo y un preservador, todo ello sellado con cinta adhesiva activada por presión. Preconizan el uso de este tipo de cintas frente a la utilización de adhesivos como el almidón de trigo o el PVA, pues son más complejos de manipular y pueden introducir humedad en el paquete daguerriano.
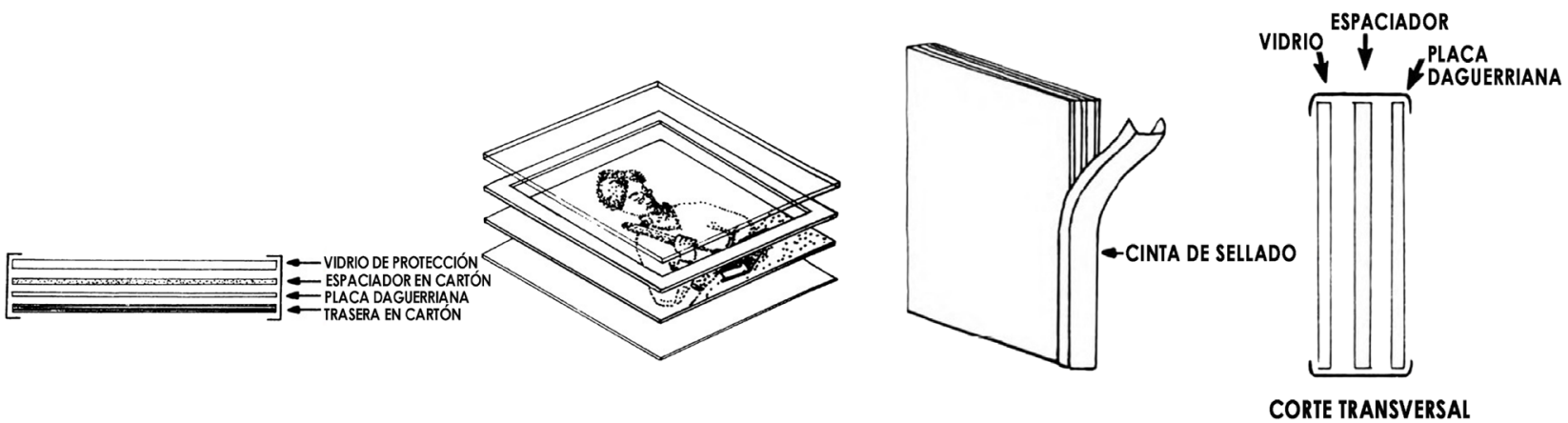

Figura 4.- Esquema de sistema de protección y resellado de placas. Dibujo por Pamela Spitzmueller en Archives \& manuscripts--administration of photographic collections (Ritzenthaler et al. 1984). Traducción: Clara M. Prieto. 
Hasta ese año, en el Metropolitan Museum of Art de Nueva York se protegían las placas sin montaje original según el diseño de Daniel Webster (MFBA 2009), que no incluía espaciador entre la placa daguerriana y el vidrio, incluía una trasera de cartón y sellaba el conjunto con cinta textil negra. A partir de entonces se incluye un espaciador y se cambia el vidrio de protección por un soporte acrílico; en el año 89 se cambia la cinta de tela por una cinta adhesiva transparente, con el fin de ampliar la visibilidad a la placa daguerriana.

En su artículo"Restoration and conservation of the Lambert gift collection of daguerreotypes" (1989) McElhone trata la intervención de una colección de daguerrotipos que, en su mayoría, se presentan montados al estilo europeo (es decir, enmarcados, con un passepartout), y su aportación constituye una valiosa fuente de información en cuanto a los materiales empleados.

De acuerdo a su artículo, el sistema de sujeción de la placa estaba formado por dos láminas de Mylar ${ }^{\circledR}-D$ (poliéster) con ventanas rectangulares de tamaño $4 \mathrm{~mm}$ menor que la placa, firmemente sujetas con tiras de cinta adhesiva de doble cara $3 \mathrm{M}^{\mathrm{TM}}$ 415. Una vez adherida, la estructura de Mylar ${ }^{\circledast}$ sujeta firmemente la placa y puede ser recortada al tamaño adecuado para su adecuación al montaje original.

Una de las principales ventajas de este sistema es que no añade prácticamente volumen al grosor final de la placa; pero presenta un importante inconveniente, no puede ser utilizado en placas que presenten exfoliación, por el riesgo de pérdida asociado al carácter electrostático del poliéster.

Otro material interesante que propone McElhone es el vidrio anti-reflectante Denglas ${ }^{\circledR}$. Este material está cubierto por ambas caras con una mezcla de óxidos metálicos que reducen el reflejo $y$, por tanto, facilitan la observación de los daguerrotipos en condiciones de iluminación no óptimas.
Respecto a los materiales celulósicos, elige cartones 100\% algodón sin reserva alcalina y como adhesivo, emplea una emulsión de acetato de polivinilo Jade 403.

En 1991 los National Archives of Canada publican "Fundamentals of photograph conservation: a study guide", compilado por Klaus Hendricks (1991). En esta obra se indica que algunos restauradores recomiendan insertar una capa intermedia entre el preservador y la placa. En concreto, recomiendan utilizar un papel (contrariamente a lo que recomienda Barger (1986)) como el papel de filtro Whatman ${ }^{\circledR}$ de $3 \mathrm{~mm}$ o bien un film de poliéster tipo Mylar $^{\circledR}$-D. Para el sellado del paquete daguerriano proponen tiras de papel japonés de alto gramaje y como adhesivo, emulsión de acetato de polivinilo, como Jade ${ }^{\circledR}$ 403, al igual que McElhone (1989).

En la citada compilación se recoge además el diseño propuesto por Swan (1981), que presenta la ventaja de poder ser instalado directamente en un marco, en el caso de su exhibición. Otra de las ventajas que se enuncian en la obra de Hendricks acerca de este montaje, es la protección que proporciona frente a los daños mecánicos y los contaminantes atmosféricos. Como quedará demostrado posteriormente en los estudios llevados a cabo por Murata (2003), dicha protección no es completamente estanca pues los sellados realizados en varias piezas son permeables a los contaminantes atmosféricos.

En un proyecto llevado a cabo en ese mismo año, la Biblioteca del Congreso de Washington procedió al montaje de una de sus colecciones de daguerrotipos, la Colección Brady-Handy (Wagner 1991).

En el montaje definitivo se emplearon cuatro materiales: vidrio de aluminiosilicato, polipropileno, Mylar $^{\circledR}$ y cinta Permacel ${ }^{\circledR} \mathrm{J}$-Lar. El vidrio se utilizó también como trasera del montaje, para permitir la observación completa de la placa daguerriana.

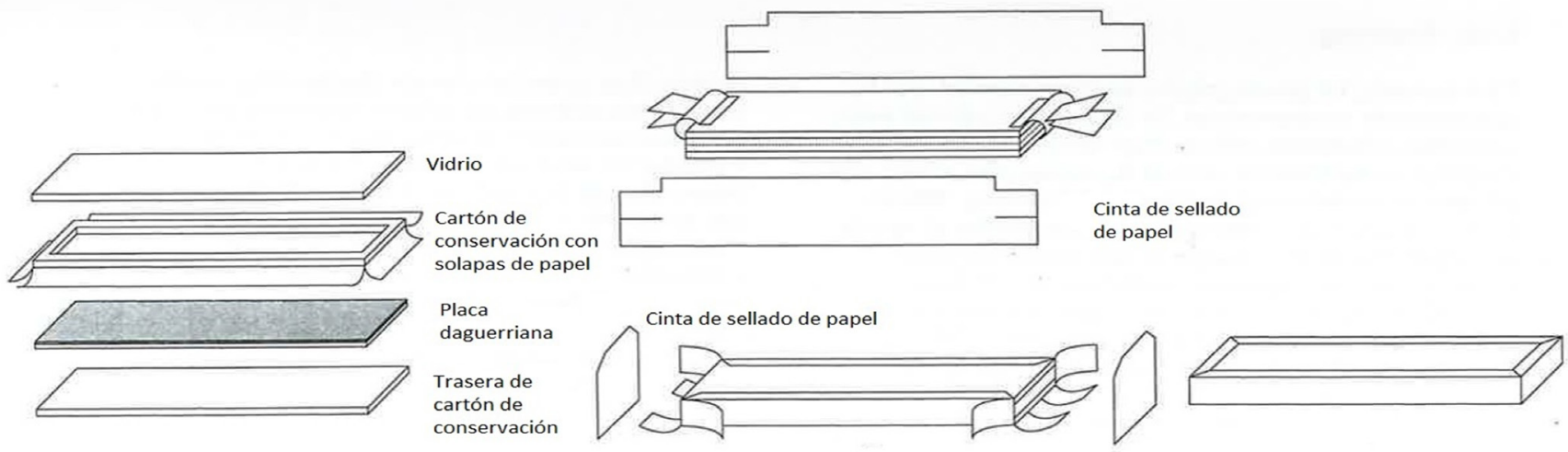

Figura 5.- Diseño elaborado por Alice Swan, (1981) y recogido por Hendricks (1991). Traducción: Clara M. Prieto. 


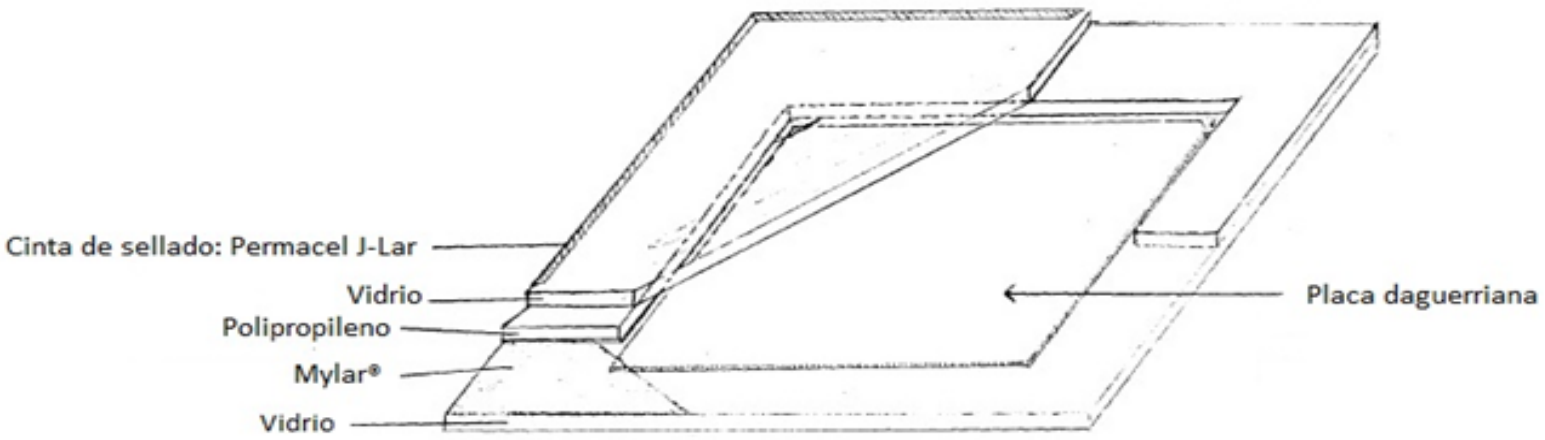

Figura 6.- Esquema del sistema de protección de placas. (Wagner 1991). Traducción: Clara M. Prieto.

Tanto el polipropileno como el Mylar ${ }^{\circledast}$ y la cinta empleada para el sellado cumplían con los requisitos del P.A.T. (Photographic Activity Test) ${ }^{6}$ y de la Norma TAPPI 444-pm 807.

En la página web de la Library of Congress se puede acceder al artículo "Preservation of the daguerreotype Collection" en el que se ofrece un esquema de sistema de montaje basado en el anteriormente descrito.

También en el año 1991, Roy Perkinson, del Museum of Fine Arts, de Boston, implementa un sistema de protección para las placas sin montaje pertenecientes a la Colección Southworth \& Hawes que profundiza en la búsqueda de la estanqueidad del sistema mediante el uso de materiales aislantes. Utiliza cartón TrueCore ${ }^{\circledR}$ para elaborar la trasera y el espaciador de la placa daguerriana (laminando dos piezas mediante cinta adhesiva de doble cara $\left.3 \mathrm{M}^{\mathrm{TM}} 415\right)$, Marvelseal ${ }^{\circledR}$ para la cubrir la trasera y los laterales del paquete, y sella el conjunto con cinta de enmarcación laminada en aluminio ${ }^{8}$. En el artículo The Collection of Southworth and Hawes Daguerreotypes at the Museum of Fine Arts, Boston, publicado en 1995 (Havinga 1995), se hace referencia a este sistema de protección. Si bien los diseños no experimentan grandes variaciones, sobre todo en lo relativo a los sistemas de sujeción de la placa daguerriana, la elección de materiales se realiza buscando la hermeticidad del sistema y avanza en función del desarrollo de la técnica.

Enelaño1995, KatrinaNewbury(MFAB2009) retomael proyecto y aplicando el mismo diseño, sustituye el cartón TrueCore ${ }^{\circledR}$, que tiene reserva alcalina, por un cartón sin ella. Las razones para esta decisión pueden deberse a las recomendaciones hechas por Ware (1994) y previamente expuestas por Collings (1980), en las que explícitamente se desaconseja el uso de papeles con reserva alcalina para el almacenamiento de fotografías.

Es en los años 90 cuando se produce un notable cambio en el diseño de los sistemas de sujeción de la placa, logrando un mínimo contacto con la misma. En la George Eastman House se desarrolla el sistema de bandejas de poliéster en Z para la sujeción de la placa en el sistema de montaje y comienza a utilizarse en sus colecciones.

En el capítulo 2 del apartado "Cased Photographs" en el Photographic Materials Conservation Catalog del Photographic Materials Group (1998) se reproduce el esquema que muestra la versión inicial del citado sistema de montaje.

La versión mejorada de dicho sistema, en uso en la actualidad, incluye una serie de modificaciones, como la reducción del tamaño de la trasera y la adaptación del vidrio al tamaño de esta. Como se puede observar en la Figura 7, el sistema en Z tiene el mínimo contacto con el anverso de la placa (que contiene la imagen) para asegurar su correcta sujeción, contactando tan solo con el borde de la misma.

En cuanto a los materiales empleados en la actualidad en la George Eastman House, según comunicación personal con Taina Meller ${ }^{9}$, se utiliza una lámina de Escal ${ }^{\mathrm{TM}}$ para elaborar las bandejas en zeta, y se sella el paquete con cinta de poliéster $3 \mathrm{M}^{\mathrm{TM}}$ o con una cinta de aluminio preparada con Filmoplast ${ }^{\circledR}$ P90, lámina de aluminio y Beva ${ }^{\circledR} 371$ en film. Si es necesario sustituir el vidrio original, se utiliza vidrio de borosilicato.

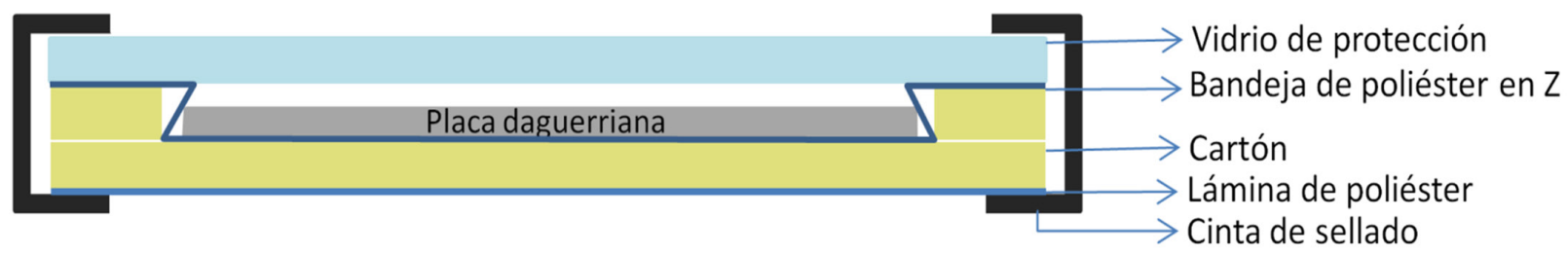

Figura 7.- Esquema del sistema de sujeción de la placa con bandejas de poliéster en zeta. Elaboración propia a partir de Murata (2003). 
En 2007, en el Weissman Preservation Center de la Universidad de Harvard, tiene comienzo el proyecto de estabilización de los 36 daguerrotipos de la Colección Agassiz del Peabody Museum of Archeology and Ethnology (Bulat et al. 2009); colección que ya había sido tratada en diversas ocasiones ${ }^{10}$. El estudio de las piezas arrojó datos muy interesantes acerca de los materiales empleados para elaborar sus sistemas de protección primaria.

De las 36 piezas, solo ocho conservaban un vidrio "histórico". El vidrio empleado en los años 90 -vidrio corriente de enmarcación- para reemplazar aquellos que presentaban deterioros avanzados resultó estar severamente dañado. Por ello, se decidió remplazar el vidrio de los 36 daguerrotipos por vidrio de borosilicato.

Respecto a las cintas de sellado, se encontraron diversos materiales: cinta autoadhesiva transparente adherida directamente al reverso de la placa; papel engomado (probablemente utilizado en el siglo XIX); papel de periódico ${ }^{11}$ adherido con un adhesivo no identificado que había perdido su función y, por último, Filmoplast ${ }^{\circledR}$
P90 (algunas piezas con trasera de papel y otras con poliéster).

Los materiales elegidos para elaborar el sistema de protección primaria fueron finalmente: Filmoplast ${ }^{\circledR}$ P90 y, en aquellos casos en que los daguerrotipos no presentaban preservador, se cubrió el Filmoplast ${ }^{\circledR}$ con una tira rehumectable de papel japonés teñido con acrílico y mica, usando como adhesivo una mezcla de Aquazol $^{\circledR}$ en agua $(85: 15)$ y añadiendo una trasera de vidrio de borosilicato. Se elige utilizar pigmentos de mica por dos razones: para imitar la apariencia de latón, puesto que el papel haría las funciones de preservador, y porque se espera que las partículas de mica ayuden a disminuir el intercambio de aire a través de la cinta de sellado, reduciendo, por tanto, la incidencia de este factor de degradación. El esquema del montaje [Figura 8] se basa en el preconizado por la George Eastman House, alojando la placa en una bandeja de poliéster con 4 zetas de sujeción y añadiendo una ventana de vidrio de borosilicato en sustitución del espaciador de cartón, con el fin de minimizar el material celulósico en el interior de los sistemas de protección primaria ${ }^{12}$.
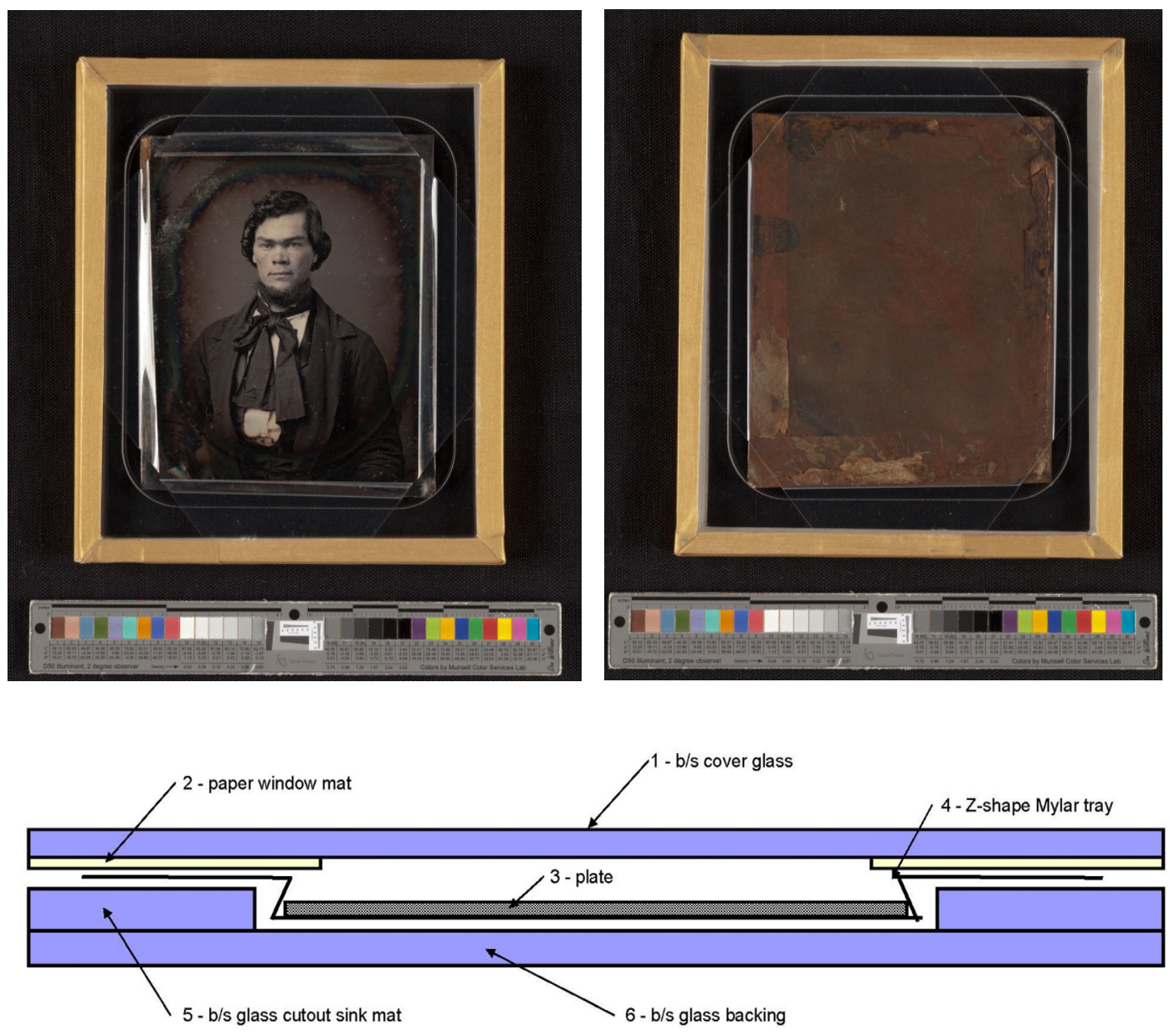

Figura 8.- Recto, verso y esquema del sistema de protección primaria implementado por Elena Bulat. Imágenes y esquema: @ Elena Bulat. 


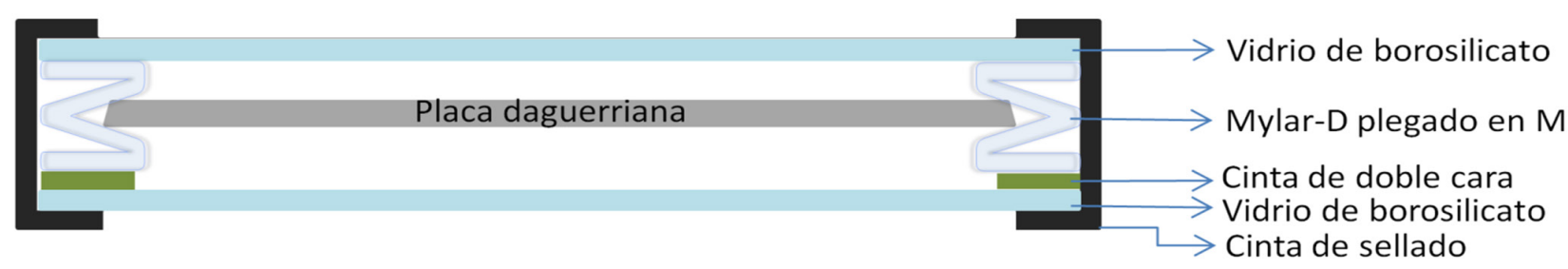

Figura 9.- Corte transversal. Esquema del diseño A. Sistema de protección primaria implementado por Chen y Beeman (2011). Traducción: Clara M. Prieto

Este sistema permite la observación íntegra de la placa daguerriana completamente protegida, por lo que da respuesta a alguna de las deficiencias encontradas en los sistemas estudiados. No obstante, presenta dificultades técnicas a priori no afrontables por instituciones modestas. La ventana de vidrio de borosilicato, por ejemplo; si bien el vidrio es un material óptimo para la función definida, no resulta sencillo de adquirir en cantidades razonables y presenta dificultades técnicas en cuanto al corte, por lo que implica que, en algunos casos, la institución debe encargar su corte a medida a terceros. Ello trae consigo la dilatación del tiempo de intervención y, por ende, el incremento de los costes del proceso, tanto por la necesidad de recursos humanos como por el coste económico de los materiales para su implementación.

Para concluir esta revisión bibliográfica, recoger las aportaciones de Chen y Beeman (2011), que proponen un sistema de protección primario para placas daguerrianas desprovistas de montaje, contenido en un sistema de protección secundario.

En el sistema de protección primaria, la placa queda suspendida entre dos vidrios de borosilicato y es mantenida por piezas de poliéster $\left(\right.$ Mylar $\left.^{\circledR}\right)$, uniendo el conjunto mediante una cinta selladora que cubre tan sólo los bordes de los vidrios, por lo que permite la completa visión de la placa daguerriana. Para el sistema de sujeción de la placa mediante piezas de poliéster proponen tres opciones de diseño. En el diseño A [Figura 9], la placa está sujeta por medio de cuatro piezas de poliéster (Mylar ${ }^{\circledast}$ ) plegadas en $M$, cortadas en bisel y aseguradas con esquineras del mismo material. En una segunda opción, las piezas de poliéster sujetan la placa en sus cuatro lados, pero sin llegar a las esquinas. En el diseño B [Figura 10], la placa descansa en una lámina de poliéster $\left(\right.$ Mylar $\left.^{\circledR}\right)$ y queda sujeta por sus bordes mediante cuatro pestañas plegadas en los lados.

De forma análoga al sistema presentado por Bulat (2009), este sistema permite la observación completa de la placa daguerriana, manteniéndola protegida. El sistema de sujeción de la placa implica un mínimo contacto con la misma y puede ser comprimido al nivel preciso, reduciendo la cantidad de aire en el interior del paquete. Siguiendo las recomendaciones de Murata (2003), la cinta de sellado se extiende sobre el vidrio, aunque deja ver la placa al completo. Asimismo, se propone un sistema de protección secundaria para contener al anterior, con el fin de limitar las interacciones químicas entre la placa daguerriana y el medioambiente.

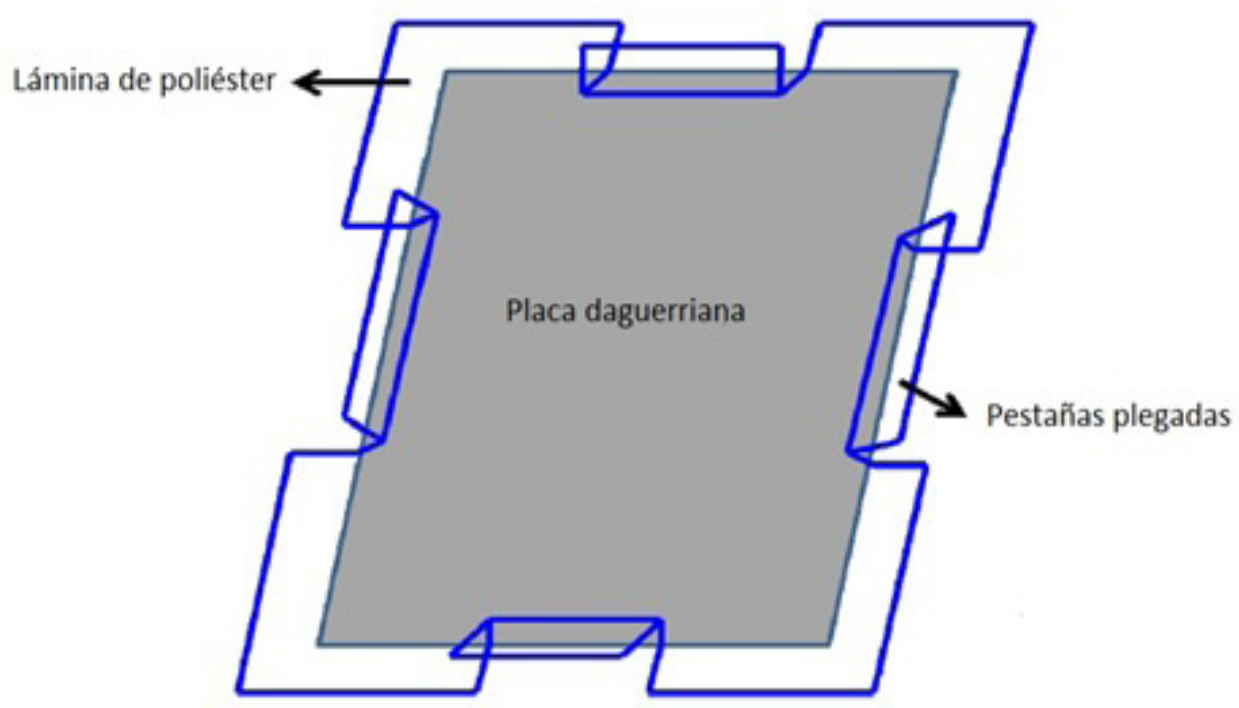

Figura 10.- Diseño B. Sistema de protección primaria implementado por Chen y Beeman (2011). Traducción: Clara M. Prieto 
Tabla 1.- Cuadro de materiales

\begin{tabular}{|c|c|c|}
\hline MATERIAL & MARCA COMERCIAL & COMPOSICIÓN (según datos del fabricante) \\
\hline \multicolumn{3}{|l|}{ ADHESIVOS } \\
\hline Acetato de polivinilo & $\mathrm{Jade}^{\circledast} 403$ & Acetato de polivinilo \\
\hline Acetato de polivinilo & Elvace $^{\circledR} 1874$ & Acetato de polivinilo \\
\hline Resina sintética & Aquazol $^{\circledR}$ & Poli (2-etil-2-oxazolina) \\
\hline \multicolumn{3}{|l|}{ PAPELES Y CARTONES } \\
\hline Papel & Papel Permalife ${ }^{\circledast}$ & 25\% de algodón. \\
\hline Papel de filtro & Whatman $^{\oplus}$ & $100 \%$ Celulosa \\
\hline Cartón & TrueCore $^{\circledast}$ & $\begin{array}{l}\text { Pulpa purificada con alto índice de alfa-celulosa, libre de } \\
\text { ácido y ligninas. Con reserva alcalina }\end{array}$ \\
\hline \multicolumn{3}{|l|}{ CINTAS ADHESIVAS } \\
\hline Cinta autoadhesiva de papel & Filmoplast ${ }^{\oplus} \mathrm{P} 90$ & $\begin{array}{l}\text { Soporte: Papel con reserva alcalina } \\
\text { Adhesivo: Acrilato }\end{array}$ \\
\hline Cinta autoadhesiva & Scotch $^{\circledast}$ Magic $^{\mathrm{TM}}$ Transparent Tape & $\begin{array}{l}\text { Soporte: Acetato de celulosa mate } \\
\text { Adhesivo: Acrilato }\end{array}$ \\
\hline Cinta adhesiva doble cara & $3 M^{\mathrm{TM}}$ DOUBLE SIDE 415 & $\begin{array}{l}\text { Adhesivo: Acrilato } \\
\text { Soporte: Poliéster }\end{array}$ \\
\hline Cinta adhesiva & Permacel $^{\circledast} \mathrm{J}$-Lar & $\begin{array}{l}\text { Adhesivo: Acrílico } \\
\text { Soporte: Polipropileno }\end{array}$ \\
\hline $\begin{array}{l}\text { Cinta de enmarcación laminada } \\
\text { en aluminio }\end{array}$ & Frame sealing tape (foil laminate) & $\begin{array}{l}\text { Adhesivo: Acrílico } \\
\text { Soporte: Papel Perma Seal, con laminado en Aluminio }\end{array}$ \\
\hline \multicolumn{3}{|l|}{ VIDRIOS } \\
\hline Vidrio antirreflectante & Denglas ${ }^{\oplus}$ antireflective glass & Vidrio con recubrimiento antirreflejo y filtro UV \\
\hline Vidrio de aluminosilicato & & $\begin{aligned} \text { Vidrio con: } & \text { Sílice }\left(\mathrm{SiO}_{2}\right): 5-60 \% \\
& \text { Óxido de Aluminio }\left(\mathrm{Al}_{2} \mathrm{O}_{3}\right): 20-40 \% \\
& \text { Óxido de Calcio }(\mathrm{CaO}): 5-50 \% \\
& \text { Óxido de Boro }\left(\mathrm{B}_{2} \mathrm{O}_{3}\right): 0-10 \%\end{aligned}$ \\
\hline Vidrio de borosilicato & & $\begin{aligned} \text { Vidrio con: } & \text { Sílice }\left(\mathrm{SiO}_{2}\right): 60-80 \% \\
& \text { Óxido de Boro }\left(\mathrm{B}_{2} \mathrm{O}_{3}\right): 10-25 \% \\
& \text { Óxido de Aluminio }\left(\mathrm{Al}_{2} \mathrm{O}_{3}\right): 1-4\end{aligned}$ \\
\hline \multicolumn{3}{|c|}{ MATERIALES DIVERSOS PARA MONTAJE } \\
\hline Polipropileno & & Copolímero de polipropileno \\
\hline Poliéster & Mylar ${ }^{\circledast}-\mathrm{D}$ & Tereftalato de polietileno \\
\hline Film barrera Aluminio & Marvelseal ${ }^{\oplus} 360$ & Polietileno, nylon y lámina de aluminio \\
\hline Film barrera transparente & $\mathrm{ESCAL}^{\mathrm{TM}}$ & $\begin{array}{l}\text { Tres capas: una exterior de polipropileno, una interior } \\
\text { de un compuesto cerámico en PVA y una interior de } \\
\text { polietileno }\end{array}$ \\
\hline
\end{tabular}


El principal inconveniente observado en este sistema es la dificultad de su aplicación práctica. Por un lado, el diseño y la elaboración de las piezas de poliéster requieren de una gran precisión en la medida y en el cálculo de las dimensiones para el plegado, resultando más sencilla la segunda opción de la versión $A$, por no implicar corte a bisel, y aún menos laboriosa la versión $B$, pues se elabora en una sola pieza.

Por otro lado, la sujeción de la placa con las piezas de poliéster es complicada $y$, en cierta medida, arriesgada, requiriendo mucha destreza, un método preciso y unas instrucciones detalladas para evitar la incorrecta y excesiva manipulación de la placa daguerriana.

La versión $B$ tiene, frente a la $A$, la ventaja de minimizar el riesgo de dañar la placa durante su montaje, pues esta descansa sobre la lámina de poliéster y las pestañas se plegarán sobre ella al mismo tiempo, en el momento de la colocación del vidrio superior.

\section{Conclusiones}

El presente trabajo ha permitido reflexionar acerca de la importancia de un adecuado sistema de protección primario para placas daguerrianas, a través de los numerosos estudios realizados al respecto y de las propuestas recogidas en la bibliografía estudiada.

La recopilación de información sobre los sistemas planteados hasta la fecha, así como las comunicaciones mantenidas con los numerosos profesionales implicados han aportado una información primordial para el estudio de los sistemas de protección primaria para daguerrotipos. El conjunto de estos datos permitirá a los custodios de este valioso patrimonio reflexionar sobre las diferentes alternativas y contribuirá a afrontar la toma de decisiones de modo informado y riguroso.

Los materiales que conforman el sistema de protección constituyen una de las principales claves en la defensa del objeto frente al medioambiente y son, por tanto, objeto de estudios actuales y futuros.

Los sistemas de protección secundaria y terciaria son considerados como complemento indispensable al sistema de protección primaria, habiendo quedado demostrada la limitada estanqueidad de este y constituyen por tanto una línea de investigación destacada.

\section{Notas}

[1] Se adopta a lo largo de este trabajo el término habitualmente empleado por Ángel Ma Fuentes de artefacto fotográfico, puesto que la palabra artefacto (RAE: Artefacto: 1. m. Obra mecánica hecha según arte.) encierra connotaciones funcionales, evocando los mecanismos, vitales en el caso de algunos especímenes fotográficos, como el daguerrotipo. La función del mecanismo es primordial en la correcta visualización del daguerrotipo, permitiendo con el juego proporcionado por la bisagra que une bandeja y cubierta obtener el ángulo adecuado de iluminación de la placa daguerriana.

[2] "archival" en el original. Según Mc Cleary (1997): "archival quality": calidad de archivo. Término usado para describir material de calidad permanente, por ejemplo, papel, cartón, etc.

Según Kosek (2004): término utilizado para referirse al papel y a otros materiales para el archivo y almacenamiento, que deben ser estables y contribuir a la preservación de estampas y dibujos conservados en ellos.

Se traduce como "papel permanente" por ser esta la definición actual dada en la Norma ISO 9706 Información y documentaciónpapel para documentos: requisitos para la permanencia (AENOR 1999) En la fecha de publicación del artículo no existía aún el comité que elaboró dicha Norma, el ISO Technical committee 46, "Information and documentation" (ISO/TC46/SC10), creado en 1998.

En la norma ISO 18902 Imaging materials-processed imaging materials, albums, framing and storage materials (ISO 2013) se establece que el término "calidad de archivo" usado como equivalente a calidad óptima de condiciones y materiales de almacenamiento, ya no es preciso. Cuando empezó a emplearse este término se asociaba a materiales de una gran durabilidad (mínimo 100 años); hoy en día se emplea ocasionalmente, incluso para duraciones estimadas de menos de 10 años. Sin embargo, la idea que continúa transmitiendo es que el material va a durar toda la vida; en vista de ello, introducen el concepto de "life expectancy" LE: expectativas de duración o duración previsible, graduándolo en "extended-term" y "medium-term" y recomiendan que los materiales no sean referidos como "calidad de archivo" sino como que cumplen con las especificaciones de esta norma internacional y de la ISO 18916 Imaging materials-Processed imaging materialsPhotographic activity test for enclosure materials (ISO 2007).

[3] Materials Reseach Laboratory (MRL). Pennsylvania State University.

[4] "Recase and seal the daguerreotype using a mat, a cover glass of modern high-durability glass, and a pressure sensitive archival paper tape to bind the package together."

[5] "Archival paper" en el original.

[6] Según la Norma ANSI IT9.2-1988. Actualmente: ISO 18916:2007 Imaging material -Processed imaging materials-Photographic activity test for enclosure materials.

[7] Silver tarnishing by paper and paperboard, Test Method. Actualmente: TAPPI/ANSI T 444 om- 12.

[8] Frame sealing tape (foil laminate) en el original.

[9] Correspondencia por e-mail, 02/04/2015.

[10] Mediante entrevistas personales se obtiene información acerca de los tratamientos practicados en los años 70, principalmente limpieza con tiourea, no existiendo entonces protocolos de 
documentación. Afortunadamente, las intervenciones practicadas en 1991, 1994, 1997 y 1999 sí fueron documentadas.

[11] Periódico de Boston de finales del S. XIX, extraordinariamente bien conservado, no presentando amarilleamiento.

[12] Esta corriente de intervención parece ser principalmente seguida por los profesionales americanos. En el ARCP (Atelier de Restauration et Conservation des Photographies de la Ville de Paris) se emplean diseños de sistemas de protección primaria similares a los estudiados, pero sustituyendo el poliéster para la elaboración de las bandejas en Z por papel, en los sistemas de protección primaria de daguerrotipos montados en passepartout (estilo europeo). Comunicación personal por e-mail, 02/04/2015, con Marsha Sirven, responsable de Restauración del ARCP. En conversaciones posteriores (08/10/2015) mantenidas durante la celebración del Daguerreobase Sysmposium (Bry-sur-Marne), se pone de manifiesto la necesidad de reflexionar acerca del modo de reducir la presencia excesiva de material celulósico en los montajes más complejos.

\section{Bibliografía}

AENOR, 1999. Información y documentación-papel para documentos: requisitos para la permanencia.UNE-EN ISO 9706:1994. Madrid: AENOR.

BARGER, M.S., 1986. "Cleaning daguerreotypes". Studies in conservation, 31, pp. 15-28.

BULAT, E., SPARTZ, I., BERNIER, B. and HOLDCRAFT, T.R., 2009. "A case for preservation: revisiting the Agassiz Collection of daguerreotypes from the Peabody Museum Archives at Harvard University". Topics in photographic preservation, 13, pp. 147-159.

CHEN, J.J. and BEEMAN, M., 2011. "Preservation System for Housing Bare Plate Daguerreotypes". Topics in photographic preservation, 14, pp. 282.

COLLINGS, T. J., 1980. Archival care of still photographs. Sheffield: Society of Archivists. Great Britain.

COLLINS, T. J.; YOUNG, F. J., 1976. "Improvements in some tests and techniques in photograph conservation". Studies in Conservation. London: Maney Publishing, pp. 79-84.

DAGUERRE, Louis Jacques Mandé. Historique et description des procédés du Daguerréotype et du Diorama (2e éd. Augm. Et Corr. Par l'Auteur). Susse Frères Éditeurs (Paris) Delloye, libraire (Paris), 1839.

DAVIES, T. L., 1977. Shoots: A Guide to Your Family's Photographic Heritage. Danbury, N.H.: Addison House.

HAVINGA, A.E., 1995. The Collection of Southworth and Hawes Daguerreotypes at the Museum of Fine Arts, Boston. The Daguerreian annual, pp. 273-274.

HENDRICKS, K.B., 1991. Fundamentals of photograph conservation: a study guide. Toronto, Ontario.: Lugus.
ISO INTERNATIONAL ORGANIZATION FOR STANDARDIZATION and TECHNICAL COMMITTEE ISO/TC-42, 2013. Imaging materialsprocessed imaging materials, albums, framing and storage materials. ISO 18902:2013. Geneva, Switzerland: ISO.

ISO INTERNATIONAL ORGANIZATION FOR STANDARDIZATION and TECHNICAL COMMITTEE ISO/TC-42, 2007. Imaging materials -- Processed imaging materials -- Photographic activity test for enclosure materials ISO 18916:2007. Geneva, Suiza: ISO.

KEEFE, L.E. e INCH, D., 1984. The life of a photograph: archival processing, matting, framing, and storage. Boston: Focal Press.

KOSEK, J.M., 2004. Conservation mounting for prints and drawings: a manual based on current practice at the British Museum. London: Archetype in association with British Museum.

LIBRARY OF CONGRESS "Preservation of the daguerreotype Collection" En http://www.loc.gov/pictures/collection/dag/ preservation.html [consulta 15/12/2015].

MCCLEARY, J.P., 1997. Conservación de libros y documentos: glosario de términos técnicos inglés-español, español-inglés. Madrid: Clan.

McELHONE, J.P., 1989. "Restoration and conservation of the Lambert gift collection of daguerreotypes". Topics in photographic preservation 3, pp. 22-27.

McELHONE, J.P. (Compiler), 1998. "Cased photographs. Photographic Materials Conservation Catalog". En: http://www. conservation-wiki.com/wiki/PMG_Cased_Photographs. [consulta $15 / 12 / 2015]$

MOORE, J. 1977, "Shoots: A Guide to Your Family's Photographic Heritage, por Thomas L. Davies", Archivaria, vol. 6.

MURATA, H., 2003. Investigation of historical and modern conservation daguerreotype housings: capstone project of Andrew W. Mellon Fellowship Advanced Residency Program in Photograph Conservation, George Eastman House.

MUSEUM OF FINE ARTS, BOSTON (MFAB), George Eastman House (GEH), Metropolitan Museum of Art (MMA), 2009. Daguerreotype Reseach Portal. En: http://research.mfa.org/. [consulta 15/12/2015].

NORRIS, Debra Hess. "The Proper Storage and Display of a Photographic Collection". The Book and Paper Group Annual. The American Institute for Conservation, 1983, vol. 2. En: http://cool. conservation-us.org/coolaic/sg/bpg/annual/v02/bp02-08.html. [consulta 15/12/2015].

PRIETO DE LA FUENTE, C. M. 2016, Embalaje de protección terciaria estructural para originales directos de cámara estuchados, Modelo de Utilidad U201500456.

REMPEL, S., 1976. "The conservation of case photographs". Archivaria, vol. 1, no. 3.

RITZENTHALER, M.L., MUNOFF, G.J. and LONG, M.S., 1984. Archives 
\& manuscripts--administration of photographic collections. Chicago: Society of American Archivists.

SWAN, A., 1978. Conservation Treatments for Photographs: A Review of Some of the Problems, Literature and Practices. En: Image 21, n 2, Rochester: International Museum of Photography at George Eastman House Inc., pp.24-31.

-1981. "The Preservation of Daguerreotypes." AIC Preprints of papers presented at the ninth annual meeting, Philadelphia, Pennsylvania. American Institute for Conservation, Washington, 1981, pp. 164-172.

TAPPI Technological Association of the Pulp and Paper Industry 2012, Silver tarnishing by paper and paperboard. Test Method. TAPPI/ANSIT 444 om-12.

THE EDITORS OF TIME-LIFE BOOKS, 1972. Cleaning a blackened daguerreotype. En: LIFE LIBRARY OF PHOTOGRAPHY, ed, Caring for photographs: display, storage, restoration. Chicago:Time-Life Books, pp. 34-39.
TIME-LIFE BOOKS, 1982. Cleaning a tarnished daguerreotype. En: LIFE LIBRARY OF PHOTOGRAPHY, ed, Caring for photographs : display, storage, restoration. Virginia: Time-Life Books INc, pp. 36-40.

WAGNER, S.S., 1991. Some recent photographic preservation activities at the Library of Congress. Topics in photographic preservation, 4, pp. 136-150.

WARE, M., 1994. Mechanisms of image deterioration in early photographs : the sensitivity to light of W.H.F. Talbot's halide-fixed images, 1834-1844. London; Bradford, West Yorkshire: Science Museum; National Museum of Photography, Film \& Television.

WEINSTEIN, R. A.; BOOTH, L., 1977. Collection, use, and Care of Historical Photographs. Nashville: American Association for State and Local History.

WIEGANDT, R., 2005. Research into the design, testing and practical application of a secondary protective housing system for daguerreotypes. Rochester: George Eastman House; Andrew W. Mellon Foundation.

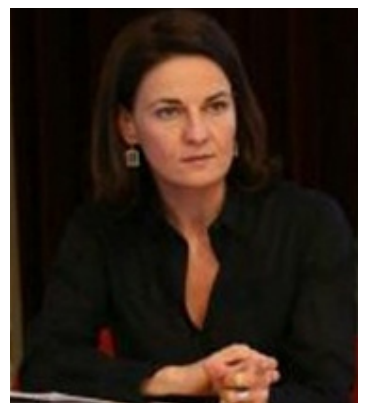

\section{Clara M. Prieto}

claramprieto@gmail.com; claramprieto@escrbc.com; info@claramprieto.com

Foto: ${ }^{\circledR}$ Carlos Vertanessian

Conservadora-restauradora independiente de fotografía y documento gráfico. Además de proyectos de investigación sobre gestión de colecciones y tratamientos de conservación, imparte clases de conservación y restauración en la ESCRBC (Escuela Superior de Conservación y Restauración de Bienes Culturales) de Madrid. Hasta 2010 trabaja para Anne Cartier-Bresson en el ARCP (Atelier de restauration et conservación des photographies de la Ville de Paris), como Responsable de proyecto (Chargée de mission) para el Plan de Salvaguarda y Valorización del Patrimonio Fotográfico (PSVPP), tras haberse especializado en conservación fotográfica en el INP (Institut National du Patrimoine), en París, Francia. Se establece en Madrid en 2010, cuando el Museo Nacional Centro de Arte Reina Sofía le concede la beca de Restauración, especialidad soporte celulósico. En 2012 estuvo becada en el Instituto de Patrimonio Cultural de España, llevando a cabo un proyecto sobre el Fondo Vaamonde. Es Máster en Conservación del Patrimonio por la UCM (Universidad Complutense de Madrid) y Titulada Superior en Conservación y Restauración de Bienes Culturales, en la especialidad de Documento Gráfico, por la ESAPA (Escuela Superior de Conservación y Restauración de Bienes Culturales de Asturias). 